\title{
Investigation of the Effect of Preoperative Hypoalbuminemia, Blood Urea Nitrogen and Creatinine Levels on Postoperative Atrial Fibrillation on Off-Pump Coronary Bypass Surgery Patients
}

\author{
Engin Akgül, Ali Ihsan Parlar, Gulen Sezer Alptekin Erkul, Sinan Erkul, Ahmet Cekirdekci \\ Department of Cardiovascular Surgery, Health Science University Kütahya Evliya Çelebi Training and Research Hospital, Kütahya, Turkey
}

\section{ABSTRACT}

Background: Postoperative atrial fibrillation (PoAF) is one of the most common complications to occur after open heart surgery. It has been shown that hypoalbuminemia accompanies some cardiovascular disorders. The present study evaluates the effects of pre-procedural albumin, blood urea nitrogen (BUN), and creatinine levels on PoAF.

Methods: The data of 81 patients who underwent offpump coronary artery bypass graft (CABG) surgery was evaluated. Patients who developed atrial fibrillation $(\mathrm{AF})$ in the first 48 hours post surgery constituted the PoAF (+) group, while those without AF constituted the PoAF (-) group. The pre-procedural hematological parameters of patients in both groups were included in the analysis.

Results: The PoAF (+) group was comprised of 57 patients $(70.3 \%)$ with a mean age of $65.5 \pm 9.8$ years, while the PoAF (-) group was comprised of 24 patients $(29.7 \%)$ with a mean age of $60.6 \pm 9.6$ years. A comparison of the demographic characteristics of the two groups showed that age $(P=.036)$, frequency of renal failure $(P=.007)$, and frequency of DM $(P=.001)$ were higher in the PoAF $(+)$ group. An examination of the laboratory data revealed a negative correlation between Hct $(P=.001)$ and albumin $(P=.000)$ levels and presence of PoAF. Also, the MPV $(P=.02), \mathrm{BUN}(P=.007)$, and $\mathrm{Cr}$ $(P=.043)$ values were higher in the PoAF ( + ) group.

Conclusion: The present study, whose focus was on the effects of albumin levels on the occurrence of PoAF, found that low levels of pre-procedural albumin, as one of the major proteins in the blood, is a risk factor for the development of PoAF.

\section{INTRODUCTION}

Coronary artery bypass graft (CABG) surgery can be considered high-risk, as the consequences concern other organs, and there is potential for a wide range of complications. With the understanding that heart and other organ perfusion

Received fuly 1, 2020; accepted fuly 15, 2020.

Correspondence: Engin Akgul, Kutahya Health Science University, Evliya Celebi Training and Research Hospital, Department of Cardiovascular Surgery, Kutahya, Turkey; +905541232827; fax +9027422366 66 (e-mail: engin_ akgul@hotmail.com). is impaired after CABG operation, measures for this were sought. Two ways became clear. The first involves performing surgery without stopping the heart, and the second involves cardioplegia, which is used to both stop the heart and prevent ischemic damage [Guajardo 2017]. In this study, we focused on operations performed on the beating heart.

Atrial fibrillation (AF) is one of the most common cardiac dysfunctions [Engin 2020]. It typically occurs 24 hours to 48 hours after surgery, although the risk of developing AF may persist until postoperative day 5. The risk of developing postoperative atrial fibrillation (PoAF) drops considerably after one week [Júnior 2015]. PoAF has been shown to cause low cardiac output, renal failure, prolonged ventilation support and needed intensive care unit, neurological events, and elevated mortality [Kowalewski 2020].

The term AF represents an incoordination between the atrium and ventricles. This lack of coordination naturally results in impairments in cardiac function and hemodynamic parameters [Ozsin 2018]. Prolonged hospitalization, thromboembolic events, heart failure, and mortality are unfavorable outcomes that can follow AF [Ozsin 2018].

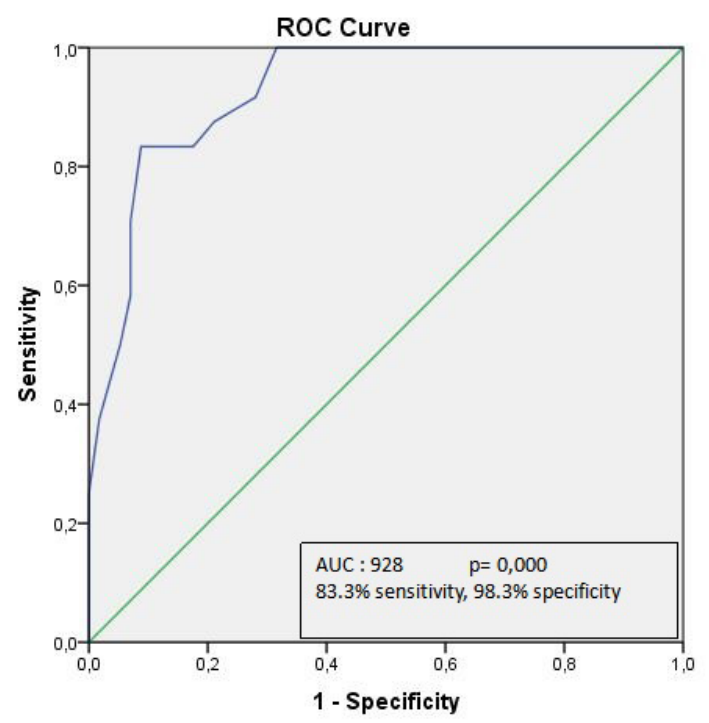

Diagonal segments are produced by ties

Receiver operation characteristic (ROC) curve and area under the curve (AUC) for albumin levels for predicting PoAF. 
Table 1. Demographic features of patients

\begin{tabular}{|c|c|c|c|}
\hline Age (years) & $60.63 \pm 9.65$ & $65.51 \pm 9.89$ & $.036 \dagger$ \\
\hline Hypertension, n (\%) & $24(51)$ & $13(41.9)$ & $.429 *$ \\
\hline Diabetes mellitus, $\mathrm{n}(\%)$ & $23(48.9)$ & $19(61.2)$ & $.001 *$ \\
\hline Number of anastomosis & $1.36 \pm 0.48(1-3)$ & $1.38 \pm 0.49(1-3)$ & $.823 \ddagger$ \\
\hline BSA & $1.79 \pm 0.15$ & $1.86 \pm 0.15$ & $.246 \dagger$ \\
\hline BMI & $26.61 \pm 4.7$ & $26.97 \pm 4.7$ & $.635 \ddagger$ \\
\hline
\end{tabular}

PoAF: postoperative atrial fibrillation, BSA: body surface area, BMI: body mass index, *Pearson Chi-Square, $\uparrow$ Student’s $t$ test, $\ddagger$ Mann-Whitney $U$ test

CABG can be performed with (on pump) or without (off pump) a cardiopulmonary bypass (CPB) machine. A heartlung machine takes over the functions of the heart in $\mathrm{CPB}$, and can therefore disrupt systemic circulation. Studies also have shown that the rate of PoAF is higher in surgical procedures performed with CPB [Engin 2020]. In another study comparing on-pump and off-pump surgeries, $\mathrm{AF}$ occurred more frequently after off-pump surgery [Kowalewski 2020]. Extracorporeal circulation may be a reason for this because blood electrolyte balance changes with extracorporeal circulation. Another possible reason is a more common development of inflammation related to surgery and global cardiac ischemia in CPB [Guenancia 2015]. Interestingly, inflammation also has been shown to increase capillary permeability. Increasing capillary permeability causes the albumin to expand to the interstitial space [Soeters 2019].

A vast number of studies have concluded that a relationship exists between low albumin levels and mortality [van Beek 2018]. This is particularly important in procedures that cause a rapid loss of albumin, such as CPB operations [van Beek 2018]. It has been reported that albumin concentrations decrease by approximately $50 \%$ with the use of priming solutions at the start of CPB surgery [Sanchez 1982; Baranyi 2012], and this decrease means that albumin levels require close follow up after surgery. We created our study from patients operated with offpump CABG. We think that not using the pump will more accurately show the relationship between preoperative albumin level and postoperative atrial fibrillation.

Albumin is the most concentrated serum protein, and it can change structure and function after glycoxidative modification [Francis 2010]. Its high solubility gives it the ability to resist many pathological events. Besides being a very powerful antioxidant, it also provides serious protection against ischemia reperfusion injury [Mapanga 2017]. Albumin also recently has been identified to be a natural angiotensinconverting enzyme inhibitor (ACE) [Fagyas 2014]. Angiotensin II (AT-II) levels decrease with the inhibition of ACE. Studies have concluded [Fagyas 2014] that albumin exerts cardioprotective effects due to its affects on AT-II, which has been shown to increase coronary resistance [Schunkert 1990] and to reduce coronary blood flow [Kozlovski 2007]. The present study, therefore, focuses mainly on whether albumin is able to prevent the development of $\mathrm{AF}$, due to its regulatory role in coronary blood flow.

It has been demonstrated that AF can develop in the presence of impairments in the kidneys, which directly affects hemodynamic status [Bansal 2013]. The rate of AF was reported to be around $20 \%$ in patients requiring dialysis or those with renal failure [Winkelmayer 2011]. Urea produced due to the breakdown of dietary protein is excreted through the kidneys, so blood urea nitrogen (BUN) can be used as an indicator of renal health. Similarly, creatinine (Cr) levels, which can be easily measured for an evaluation of renal function, also indicate the status of kidneys. Renal failure causes electrolyte imbalance, which is the one of the atrial fibrillation causes. Also, higher cardiovascular events have been shown when creatinine clearance is $<80 \mathrm{~mL} / \mathrm{min}$ [Kodani 2018].

Platelet activation leads to platelet pseudopodia formation as well as changes in the morphology and distribution that result in a spheric appearance. Accordingly, the mean platelet volume (MPV) and platelet distribution width (PDW) are easily identified and are useful sources of information about the platelets. Platelets form a larger structure upon activation, and their prothrombotic factor and vasoactive substance concentrations increase. These prothrombotic and vasoactive substances explain why platelets play a central role in thrombotic and inflammatory conditions. Likewise, increases in the MPV during thrombotic cardiovascular and cerebrovascular events have been shown to be related to mortality [Providência 2013].

\section{MATERIALS AND METHODS}

Patients: Included in this retrospective study were 81 patients who underwent off-pump cardiopulmonary bypass grafting surgery between 2013 and 2019. The study was granted approval by the local institutional Ethics Committee of the University of Health Sciences (2019/88, 30.09.2019).

The exclusion criteria for this retrospective study were presence of preoperative $\mathrm{AF}$ or atrial flutter, preoperative 
Table 2. Laboratory variables of the patients

\begin{tabular}{|c|c|c|c|}
\hline Hematocrit (\%) & $40.06 \pm 3.65$ & $37.45 \pm 3.25$ & $.001 \ddagger$ \\
\hline Platelet $\left(10^{3} / \mu \mathrm{L}\right)$ & $229.6 \pm 127.6$ & $193.7 \pm 67.3$ & $.154 \dagger$ \\
\hline Mean platelet volüme $(\mathrm{fL})$ & $8.83 \pm 1.1$ & $9.23 \pm 1.34$ & $.020 \ddagger$ \\
\hline Creatinine $(\mathrm{mg} / \mathrm{dL})$ & $1.04 \pm 0.24$ & $1.37 \pm 1.02$ & $.043 \ddagger$ \\
\hline Free $\mathrm{T}_{4}(\mathrm{ng} / \mathrm{dL})$ & $1.18 \pm 0.32$ & $1.04 \pm 0.09$ & $.186 \dagger$ \\
\hline $\mathrm{TSH}(\mathrm{IU} / \mathrm{mL})$ & $3.32 \pm 5.20$ & $2.43 \pm 1.41$ & $.884 \dagger$ \\
\hline Albumin (g/dL) & $3.77 \pm 0.47$ & $2.87 \pm 0.34$ & $.000 \dagger$ \\
\hline
\end{tabular}

BUN: blood urea nitrogen, TSH: thyroid stimulating hormone, †Student’s $t$ test, $\ddagger$ Mann-Whitney $\cup$ test

amiodarone use, presence of valvular heart disease, and redo operations. The demographic characteristics of the patients also were examined.

Laboratory measurements: The presence of diabetes, renal failure and other additional pathological conditions was determined through analyses of fasting blood samples collected in EDTA tubes as part of preoperative preparation. The blood samples were analyzed using an automated hematological analyzer (Coulter LH 780 Analyzer, CA, USA).

Diagnosis of PoAF: Patients were moved to the intensive care unit after surgery while the ET tube was in place, and the patients were intubated until muscle strength returned to normal. The hemodynamic parameters of the patients were monitored via continuous invasive blood pressure measurement and cardiac rhythm monitorization. In addition, cardiac rhythm was documented with a daily 12-lead ECG recording, and a further 12-lead ECG was recorded upon the emergence of any extraordinary complaints. Medical cardioversions were performed after confirming the abnormal rhythms observed on the monitor to be associated with $\mathrm{AF}$, involving the administration of amiodarone $(5 \mathrm{mg} / \mathrm{kg})$. A loading dose was administered over a 30-minute period, followed by a maintenance dose of $900 \mathrm{mg} /$ day.

Statistical analysis: The Statistical Package for Social Sciences (IBM SPSS Statistic Inc. version 21.0, Chicago, IL, USA) software was used for statistical analysis. Nominal variables were expressed as frequency and percentages, and continuous variables were expressed as mean \pm standard deviation. Kolmogorov-Smirnov and Shapiro-Wilk normality tests were used to evaluate the distribution of the variables. A Student's $t$-test was used to compare continuous variables with normal distribution, and a Mann-Whitney $\mathrm{U}$ test was used to compare variables without normal distribution. A $P$-value less than 0.05 was considered statistically significant in all tests. A receiving operating characteristic (ROC) curve analysis was performed to determine the variables predicting the development of PoAF after off-pump CABG surgery, and the area under the curve was calculated for albumin levels.

\section{RESULTS}

Many hematological parameters change due to the fact that the blood flows into the extravascular area in on-pump CABG. While planning this study, external factors that may affect the results were reduced as much as possible. So, onpump surgeries were excluded from this study to achieve more reliable results.

The present study compared the development of PoAF in 81 patients after off-pump cardiopulmonary bypass graft surgery. The PoAF (+) group included 57 patients $(70.03 \%)$ with a mean age of $60.6 \pm 9.6$ years, and the PoAF (-) group included 24 patients $(29.7 \%)$ with a mean age of $65.51 \pm$ 9.89 years. When evaluating gender, it was seen that the PoAF (-) group had more male patients $(60 \%)$ and PoAF $(+)$ had more female patients $(52 \%)$. However, this finding was not statistically significant. An analysis of the demographic characteristics of the patients in the two groups, as presented in Table 1 , revealed statistically significant differences in terms of age $(P=.036)$, renal failure $(P=.007)$, and $\mathrm{DM}(P=.001)$.

From the laboratory data, a negative correlation was identified between hematocrit (Hct) and albumin levels and the presence of PoAF. PoAF often was accompanied by low Hct $(P=.001)$ and albumin $(P=.000)$ values. Furthermore, a positive correlation was observed between the presence of PoAF and MPV $(P=.02), \mathrm{BUN}(P=.007)$, and $\operatorname{Cr}(P=.043)$ levels. So, elevated preoperative MPV values, $\mathrm{BUN}$, and $\mathrm{Cr}$ levels are a predictor for PoAF (Table 2).

Finally, the results of the ROC curve analysis for albumin was as follows: a cut-off level of 3.05 for predicting PoAF (area under the curve (AUC): 0.925, 95\% CI: $0.873-0.982, \log$ rank $P=.000,83.3 \%$ sensitivity, $98.3 \%$ specificity) (Figure). These findings show us there are some changeable parameters, such as low albumin and Hct levels, causing PoAF and we can take action against this situation. 


\section{DISCUSSION}

$\mathrm{AF}$ is one of the most common complications to occur after open heart surgery, with reported incidence rates in the range of $20-40 \%$ [Phan 2015]. The inability of the atria to effectively contract decreases cardiac output over time by impairing ventricular function, thus worsening the symptoms of heart failure and resulting in thromboembolism, stroke, and sudden death [Camm 2010]. Accordingly, many studies have been conducted into AF. The present study evaluates the effect of albumin levels on the development of AF. A statistical analysis revealed that the rate of $\mathrm{AF}$ was significantly higher in cases with low albumin levels $(P=.000)$.

Albumin is the natural transport protein in blood and has a long half-life. It is an antioxidant and also a marker of inflammation [Chien 2017]. Albumin may exert its effect on the development of AF by decreasing AT-II levels, and may thus lead to coronary ischemia, while its antioxidant effects may be associated with its ability to retain the fluid in the intravascular area and prevent the development of hypovolemia.

Hypoalbuminemia is common in the post-cardiac surgery period and especially in elderly patients with chronic heart failure [Novack 2010]. Many studies have concluded that low albumin is associated with cardiac mortality and morbidity. In some studies, hypoalbuminemia has been shown to cause a pulmonary congestion [Arques 2011], myocardial edemaand myocardial dysfunction [Dongaonkar 2010], diuretic resistance and fluid retention [Elwell 2003], decrease in antioxidant functions and anti-inflammatory properties [Roche 2008].

In the present study, a high rate of $\mathrm{AF}$ was observed among patients with renal failure $(P=.007)$. In addition, BUN and Cr levels were higher in the PoAF $(+)$ group $(P=.007$ and $P=.043$, respectively). Renal insufficiency (RI) paves the way for many other diseases that can threaten the patient's life, although studies also have shown that RI alone may result in the development of AF [Okutucu 2017]. This can be attributed to the electrolyte imbalance that occurs in RI, acid-base disturbances, hypertension, and left atrial and left ventricular dilation occurring in time in patients with RI [Allison 2013]. The results of the present study are consistent with those reported in literature.

Advanced age is a widely accepted cause of PoAF [Greenberg 2017]. A decrease in myocardial fibers and fibrosis, and collagen accumulation around the atrium and synoatrial nodes occur over time, and the incidence of PoAF thus increases with age [Greenberg 2017]. According to a study of 14,960 patients who underwent cardiac surgery, the risk of developing PoAF increases after age 55, and the risk is five-fold higher after age 72 [Shen 2011]. The mean age in the PoAF $(+)$ group $(65.51 \pm 9.89$ years $)$ in the present study was higher than in the other group $(P=.036)$.

$\mathrm{AF}$ has been shown to occur frequently in patients with diabetes mellitus. In the VHAH study, DM was shown to be an independent and strong risk factor for the development of AF [Movahed 2005]. The oxidative stress and connexin remodeling observed in diabetic patients can be suggested as the cause of myocardial apoptosis, lipotoxicity, hypertrophy, metabolism disorders and decreases in contractile capacity in patients exhibiting symptoms of diabetic cardiomyopathy [Movahed 2005; Goudis 2016]. In the present study, DM was observed in $61.2 \%$ of patients in the PoAF (+) group, which was significantly higher than in the PoAF $(-)$ group $(P=.001)$. These findings are consistent with literature.

The Hct value is the ratio of the volume of hemoglobin in the blood to the total volume of blood, and the relationship between Hct values and PoAF was investigated in the present study. The statistical analysis showed that Hct values were lower in the PoAF $(+)$ group $(P=.001)$. PoAF is more likely to develop in patients with low Hct levels [Öztürk 2018]. Low hemoglobin values as a risk factor for the development of PoAF can possibly be attributed to the low supply of oxygen to the myocardium, although the fact that this does not apply to low Hct values can be the subject of another study.

MPV is one of the parameters that commonly has been associated with the development of AF. There have been many studies conducted into this subject in which the relationship between the two parameters has been linked to the vasoactive substances released by the platelets during inflammatory conditions and the morphological changes that the platelets undergo. There have been studies reporting a higher risk of AF during the inflammatory period [Aviles 2003]. Increased inflammation means high MPV, which translates into low coronary blood flow [Feng 2013], more frequent thromboembolic events and left atrial stasis [Feng 2013]. In the present study, MPV was significantly higher in the PoAF (+) group $(P=.020)$, and this finding is consistent with those of previous studies.

Albumin has been the main focus of the present study. Patients with valvular disease and those with obstructive lung disease were excluded from the study. As predicted, albumin levels were found to be associated with PoAF.

\section{CONCLUSION}

One of the most researched topics undoubtedly is atrial fibrillation. A wide range of studies of PoAF have been carried out, evaluating DM, HT, RI, MPV, RDW, PLT and similar factors. While it can be treated, PoAF also can lead to serious complications. The present study found preoperative low albumin and Hct levels to be a risk factor for PoAF. Both parameters are easily replaceable before the operation, and this is easy way to prevent AF. In addition, considering the functions of albumin in the body, it is understood that hypoalbuminemia and low Hct should be treated more aggressively during the postoperative period.

There were some limitations to this study. This was a single center and retrospective study. Off-pump CABG surgeries were not performed by a single surgeon. The number of patients included in the study is small. And finally, there is no record of long-term outcomes. 
REFERENCES

Allison SJ. 2013. Chronic kidney disease: ESRD risk in CKD patients with incident atrial fibrillation Nat Rev Nephrol 9:125.

Arques S, Ambrosi P. 2011. Human serum albumin in the clinical syndrome of heart failure. J Card Fail. 17:451-458.

Bansal N, Fan D, Hsu CY, Ordonez JD, Marcus GM, Go AS. 2013. Incident atrial fibrillation and risk of end-stage renal disease in adults with chronic kidney disease Circulation. Feb 5;127(5):569-74.

Baranyi A, Rothenhäusler H-B. 2012. The impact of intra- and postoperative albumin levels as a biomarker of delirium after cardiopulmonary bypass: results of an exploratory study. Psychiatry Res. 200(2-3):957-963.

Camm J, Kirchhof P, Lip GYH, Schotten U, Savelieva I, Ernst S, et al. 2010. Guidelines for the management of atrial fibrillation: the Task Force for the Management of Atrial Fibrillation of the European Society of Cardiology (ESC) Eur. Heart J., 31 pp. 2369-2429.

Chien SC, Chen CY, Lin CF, Yeh HI. 2017. Critical appraisal of the role of serum albumin in cardiovascular disease Biomark Res. Nov 10;5:31.

Dongaonkar RM, Stewart RH, Geissler HJ, et al. 2010. Myocardial microvascular permeability, interstitial oedema, and compromised cardiac function. Cardiovasc Res. 87:331-339.

Elwell RJ, Spencer AP, Eisele G. 2003. Combined furosemide and human albumin treatment for diuretic-resistant edema. Ann Pharmacother. $37: 695-700$.

Engin M, Aydın C. 2020. Investigation of the Effect of HATCH Score and Coronary Artery Disease Complexity on Atrial Fibrillation After OnPump Coronary Artery Bypass Graft Surgery Med Princ Pract. May 18.

Erdolu B, As AK, Engin M. 2020. The Relationship between the HATCH Score, Neutrophil to Lymphocyte Ratio and Postoperative Atrial Fibrillation After Off-Pump Coronary Artery Bypass Graft Surgery. The Heart Surgery Forum 23(1); 88-92.

Fagyas M, Úri K, Siket IM, et al. 2014. New Perspectives in the ReninAngiotensin-Aldosterone System (RAAS) II: Albumin Suppresses Angiotensin Converting Enzyme (ACE) Activity in Human. Karamyan V, ed. PLoS One. 9(4):e87844.

Feng C, Mei W, Luo C, et al. 2013. Relationship between mean platelet volume and coronary blood flow in patients with atrial fibrillation. Heart Lung Circ. 22:43-9.

Francis GL. 2010. Albumin and mammalian cell culture: implications for biotechnology applications. Cytotechnology 62:1-16.

Goudis CA, Korantzopoulos P, Ntalas IV, Kallergis EM, Liu T, Ketikoglou DG. 2015. Diabetes mellitus and atrial fibrillation: Pathophysiological mechanisms and potential upstream therapies Int J Cardiol. Apr $1 ; 184: 617-22$.

Greenberg JW, Lancaster TS, Schuessler RB, Melby SJ. 2017. Postoperative atrial fibrillation following cardiac surgery: a persistent complication Eur J Cardiothorac Surg. Oct 1;52(4):665-672.

Guajardo Salinas GE, Nutt R, Rodriguez-Araujo G. 2017. Del Nido cardioplegia in low risk adults undergoing first time coronary artery bypass surgery. Perfusion. 32(1):68-73.

Guenancia C, Pujos C, Debomy F, Malapert G, Laurent G, Bouchot O. 2015. Incidence and Predictors of New-Onset Silent Atrial Fibrillation after Coronary Artery Bypass Graft Surgery. Biomed Res Int 2015:703685.

Júnior MSB, Matkovski PD, Di Giovanni FJ, Fenili R, Varella EL,
Dietrich A. 2015. Incidence of postoperative atrial fibrillation in patients undergoing on-pump and off-pump coronary artery bypass grafting. Braz J Cardiovasc Surg 30:316-24.

Kodani E, Atarashi H, Inoue H, et al. 2018. Impact of creatinine clearance on outcomes in patients with non-valvular atrial fibrillation: a subanalysis of the J-RHYTHM Registry. Eur Heart J Qual Care Clin Outcomes. 4(1):59-68.

Kowalewski M, Jasiński M, Staromłyński J, et al. 2020. On-Pump vs OffPump coronary artery bypass surgery in atrial fibrillation. Analysis from the polish national registry of cardiac surgery procedures (KROK) PLoS One 15(4):e0231950.

Kozlovski VI, Lomnicka M, Jakubowski A, Chlopicki S. 2007. Inhibition of neutral endopeptidase by thiorphan does not modify coronary vascular responses to angiotensin I, angiotensin II and bradykinin in the isolated guinea pig heart. Pharmacol Rep. 59(4):421-427.

Mapanga RF, Joseph DE, Saieva M, et al. 2017. Glycation abolishes the cardioprotective effects of albumin during ex vivo ischemia-reperfusion. Physiol Rep. 5(2):e13107.

Movahed R, Hashemzadeh M, Jamal MM. 2005. Diabetes mellitus is a strong, independent risk for atrial fibrillation and flutter in addition to other cardiovascular disease, Int. J. Cardiol. 105 315-318.

Novack V, Pencina M, Zahger D, et al. 2010. Routine laboratory results and thirty day and one-year mortality risk following hospitalization with acute decompensated heart failure. PLoS One. 5:e12184.

Okutucu S, Öztürk D, Oto E, Güvenir HA, Karaagaoglu E. 2017. Data mining experiments on the angiotensin II- Antagonist in Paroxysmal Atrial Fibrillation (ANTIPAF - AFNET 2) trial: 'exposing the invisible' Europace. May 1;19(5):741-746.

Ozsin KK, Sanri US, Toktas F, Yavuz S. 2018. Relationship between red cell distribution width and mean platelet volume with new onset atrial fibrillation after off-pump coronary artery bypass grafting Bratisl Lek Listy. 119(6):335-34.

Öztürk İ, Öztürk S. 2015. Association between postoperative atrial fibrillation and the levels of hemoglobin or hematocrit: a systematic review and metaanalysis Anaesthesia, Pain \& Intensive Care Jul 19; 3: 247-253.

Phan K, Ha HS, Phan S, Medi C, Thomas SP, Yan TD. 2015. Newonset atrial fibrillation following coronary bypass surgery predicts longterm mortality: a systematic review and meta-analysis Eur J Cardiothorac Surg. Dec;48(6):817-24.

Providência R, Faustino A, Paiva L, et al. 2013. Mean platelet volume is associated with the presence of left atrial stasis in patients with nonvalvular atrial fibrillation. BMC Cardiovasc Disord. 10;13:40.

Roche M, Rondeau P, Singh NR, et al. 2008. The antioxidant properties of serum albumin. FEBS Lett. 582:1783-1787.

Sanchez de Léon R, Paterson JL, Sykes MK. 1982. Changes in colloid osmotic pressure with plasma albumin concentration associated with extracorporeal circulation. BrJAnaesth. 54(4):465-473.

Schunkert H, Dzau VJ, Tang SS, Hirsch AT, Apstein CS, Lorell BH. 1990. Increased rat cardiac angiotensin converting enzyme activity and mRNA expression in pressure overload left ventricular hypertrophy. Effects on coronary resistance, contractility, and relaxation. J Clin Invest. 86(6):1913-1920.

Shen J, Lall S, Zheng V, Buckley P, Damiano RJ, Jr Schuessler RB. 2011. The persistent problem of new-onset postoperative atrial fibrillation: a single-institution experience over two decades J Thorac Cardiovasc Surg 141:559-70. 
Soeters PB, Wolfe RR, Shenkin A. 2019. Hypoalbuminemia: Pathogenesis and Clinical Significance. JPEN J Parenter Enteral Nutr. 43(2):181-193.

van Beek DEC, van der Horst ICC, de Geus AF, Mariani MA, Scheeren TWL. 2018. Albumin, a marker for post-operative myocardial damage in cardiac surgery J Crit Care. Oct;47:55-60.

Winkelmayer WC, Patrick AR, Liu J, Brookhart MA, Setoguchi S. 2011. The increasing prevalence of atrial fibrillation among hemodialysis patients. J Am Soc Nephrol. 22:349-357. 